Grant ivo

$F(602-86 L R / 34 \%)$ ?QNFE $90116-4.3$

International Conference on the Application of

Accelerators in Research and Industry

Denton, Texas

November $5-8,1990$

CONF-901116--43

DE93 006815

\title{
The Physics of Electron Beam Ion sources
}

\author{
Martin P. Stockli and C.L. Cocke \\ J.R. Macdonald Laboratory, Kansas State University \\ Manhattan, KS 66506-2604, USA
}

\section{Abstract}

There are 13 Electron Beam Ion Sources in operation which produce highly charged ions, up to $\mathrm{Th}^{80+}$ and $\mathrm{Xe}^{53+}$. Most of the sources are used to study these ions under electron impact or when recombining with gaseous or solid targets. That provides an insight into the atomic physics of these highly charged ions and into the physics of the plasma in which such ions can be found. This paper reviews the present knowledge of atomic processes, important in the production of such ions with an EBIS.

\section{MASTER}




\section{Introduction}

The concept of an Electron Beam Ion Source (EBIS) for the production of highly charged ions was proposed by Donets in 1968 [1]. Today, more than twenty years later, there are a total of

13 operational EBISes located in Russia, Western Europe, the United States, and Japan [2]. Eight of those EBISes produce fully stripped Argon on a standard basis and much higher charge states for heavier ions. Recently, the Livermore group published a magnetically separated charge state distribution of Uranium, which clearly demonstrates the production of $\mathrm{U}^{70+}[3]$. Even more recently the group broke its own record with the production of neonlike Thorium, $\operatorname{Th}^{80+}[4]$. However, the highest ionization energy was achieved by Donets and coworkers with the successful production of hydrogenlike Xenon [5-7]. These are the highlights of the recent progress of the EBIS development and more details can be found in the proceedings of the 1988 EBIS Symposium [8] and of the 1989 Ion Source Conference [9]. EBISes produce such highly charged ions at a typical potential of one to a few kilovolts and therefore the ions can be extracted to form ion beams. The low emittance and the small energy spread of the extracted ions minimize losses during transport, which is accomplished with standard ion optical elements for low rigidity charged particles. The energy of the ions can be varied with the potential of the production site, which goes from a range of a few kilovolts (limited by a typical trap power supply) up to 200 $\mathrm{kV}$ if a high voltage platform is used [10]. No other ion source or accelerator system can deliver beams of such highly charged 
ions in this low energy range. Four of the EBISes are designed as or working as injectors for synchrotrons; some are used to test EBIS technology, but most of these ion sources are used to explore the atomic physics of highly charged ions. Some of the Iatter EBISes, especially the Livermore EBIT [11], are used as Electron Beam Ion Traps to study the electron-ion interaction inside the source and keep producing unique information on electron impact ionization, excitation, and recombination of highly charged ions. However, the bulk of the latter EBISes, which since recently includes Livermore's EBIT [3], deliver their ions to remote experimental stations in which the ions can interact with other matter or radiation. So far, the published results, as well as the experiments underway, concentrate on high yielding experiments, such as the recombination of the highly charged ions colliding with atoms or solids. Clearly, the EBISes contribute substantially to the exploration of the physics of highly charged ions and to the physics of the plasma, in which such ions can be found. In this paper, we review briefly the present understanding of the atomic processes which play an important role in the production of highly charged ions with an EBIS.

\section{The Evolution of the Charge states}

The dominant component of a typical EBIS is its high field, nowadays mostly superconducting solenoid, as shown in Figure 1 . Also shown is the electron gun, mounted on the axis at the entrance of the solenoid. The electron beam is compressed as it 
enters the increasing magnetic field and expands with the decreasing magnetic field as it exits, where the beam is absorbed inside a collector. The axial exit hole in the collector allows the ions to exit, while the repeller repels the axial electrons. The potential of the electron beam is determined by a series of drift tubes which allows one to adjust and alter the potential profile. EBISes are seeded with a gas bleed or with low charged ions injected from an internal preionization stage or from an external ion source. Once inside the solenoid, the ion is trapped radially by the Lorentz force and by the space charge of the electron beam. The electrostatic potential at both ends of the trap can be raised to form a potential barrier. When the potential of such a barrier is lowered, or the central trap potential is raised, or the ions gain sufficient energy, they will escape over the barrier. If the injection of low charged ions, followed first by a containment for a certain time $\tau_{c}$, and then by the ejection of the ions, is constantly repeated, we have a pulsed EBIS. In this paper we concentrate on this mode of operation, for which the confinement time $\tau_{c}$ is obviously well known.

A trapped ion is subject to continuous bombardment with fast electrons which strip the ion, electron by electron. Neglecting multiple ionization, the evolution of the charge states can be calculated with the follo ng set of differential equations for the population of the charge state $n_{q}$ :

$$
\frac{d n_{q}}{d(j \cdot t / e)}=n_{q-1}(t) \cdot \sigma_{i}(q-1, q)-n_{q}(t) \cdot \sigma_{i}(q, q+1)
$$


where $e$ is the electron charge, and $j \cdot t$, the product of the electron beam current density and the bombarding time, traditionally called the ionization factor, is proportional to the number of beam electron-ion collisions. The cross section for the electron impact ionization of the qth electron $\sigma_{i}(q-1, q)$ is normally approximated with the cross section for direct ionization, which has a threshold at ionization energy $I_{q-1}$ of the qth electron, and reaches a maximum at 2-3 times $I_{q-1}$, then decreases slowly with increasing electron energy, for which exist many simple approximations [12]. The solution of (1) for a pulsed EBIS with only neutrals at $t=0$ yields the evolution of the charge states, as one calls the changing charge state distribution as a function of the ionization factor, shown in Figure 2 for Argon [from 12]. However, normally the approximations of the electron impact ionization underestimate the true cross sections for many-electron ions due to the importance of indirect ionization [13]. In addition, many electron ions exhibit a substantial amount of multiple ionization at higher impact energies [14], and therefore the calculated charge state distributions are not very accurate for many-electron ions [15]. However, the EBIS is normally used to produce ions with no or a few remaining electrons, a process dominated by the removal of the last few electrons with an electron beam energy approximately twice the ionization energy of the last electron to be ionized. Since direct ionization is the dominant process for the removal of the last few electrons, this simple model normally describes the observed charge state 
distributions in a reasonable fashion, as one can conclude from Figure 3 [from 16], which shows the charge state distributions of Argon obtained for 4 different confinement times. Using the charge state distribution at $\tau_{c}=0.04$ seconds, one determines from Figure 2 a $j \cdot t$ of approximately 26, yielding a current density of approximately $650 \mathrm{amps} / \mathrm{cm}^{2}$, which would be very difficult to measure directly inside an EBIS. And as a matter of fact, the evolution of charge states also can be used to extract electron impact ionization cross sections for highly charged ions $[5,17,18]$

If one is interested only in the average charge state <q>, we can add, starting with the initial charge state $i$, all average ionization factors $j \cdot\left\langle t_{q}\right\rangle$ required to remove the $q$ th electron and therefore obtain the total ionization factor $j \cdot\left\langle\tau_{a}\right\rangle$ to reach the average charge state $\langle Q\rangle$ :

$$
\frac{j \cdot\left\langle\tau_{Q}\right\rangle}{e}=\sum_{q=i+1}^{0} \frac{1}{\sigma_{i}(q-1, q)}
$$

This approximation is often used to estimate the ionization factor or confinement time required to produce a certain charge state $Q[5,7,19,20]$. This approximation implies that any charge state $Q$ could be obtained, if the electron beam energy is above the ionization energy of the last electron $I_{Q-1}$ to be removed, and If the ion is bombarded for a sufficiently long time, $\tau_{a}$.

However, the highest achievable charge state is often limited by recombination processes which are discussed in the next section. 


\section{The Recombination of Ions}

Recombination of the ions occurs when they capture electrons in collisions with free electrons, other ions, or neutrals. A free electron can be captured through radiative recombination in which electromagnetic radiation carries away the excess energy. The cross section for an ion with charge state $q$ to capture an electron into a hydrogenic orbital with the main quantum number $n$ was derived as

$$
\sigma_{n}=2.1 \cdot 10^{-22} \cdot \frac{q^{4} \cdot E_{o}^{2}}{n \cdot E_{\theta} \cdot\left(q^{2} \cdot E_{o}+n^{2} \cdot E_{\theta}\right)}
$$

where $E_{0}$ is the Rydberg energy and $E_{e}$ the energy of the free electron [21]. Clearly, this process has a small probability, especially for the fast beam electrons. It was, however, observed with EBIT [22]. Radiative recombination with low energy electrons is much more likely and has recently been measured in the energy range between 0 and $1 \mathrm{eV}$ [23]. The secondary electrons, which are released from the ions during ionizing collisions, can feature low energies, depending on the primary electron energy and the impact parameter. However, such low energy electrons are rapidly heated by collisions with fast electrons, and therefore will leave the electron beam and eventually the trap.

s primary electron colliding with a partially stripped ion may not ionize, but only promote a bound electron into an excited state. This electron impact excitation also has been measured in 
EBISes by observing the x-rays emitted during deexcitation $[22,24-26]$. In this process, the primary electron loses energy equal to the difference between the excited and the ground state of the excited electron. In fact the primary electron can lose more than its initial energy and end up in a bound state of the target ion. Since the final energy of the primary electron must coincide with one of the ion's discrete levels, we have a resonant process, which only works if the initial energy of the primary ion is equal to the level difference gained by the bound electron minus the ionization energy of the final bound state of the primary electron. This process, which forms doubly excited states, is called dielectronic recombination and several measurements have been performed with EBISes [24,26-29]. Figure 4 [from 26] shows the dielectronic recombination cross section for heliumlike Argon, which is essentially the ratio between the lithiumlike and the heliumlike ion yields multiplied by the ionization cross section for the lithiumlike ion [28]. This result was obtained from the measured yields of extracted heliumlike and lithiumlike Argon ions, after they were bombarded by the electron beam for 2 seconds to reach the equilibrium charge state distribution. This measured equilibrium charge state distribution is shown in Figure 5 [from 26] as a function of the electron beam energy, which clearly indicates the importance of dielectronic recombination for the production of not fully stripped ions. Destructive recombination can be minimized by choosing an electron beam energy which is below the threshold for dielectronic recombination, or in between 
resonances, or above the kLn series limit. It is interesting to compare Figure 5 with Figure 6 [from 26] which shows the K-X-ray production as a function of the electron beam energy. Electron impact excitation with an energy above the excitation threshold produces $x$-rays but naturally no change of charge, and so do the higher doubly-excited dielectronic resonances, because they obviously autoionize.

When an ion with a charge state q collides with another ion, atom, or molecule, a bound electron from the collision partner may be released, if this electron senses a force from the incident ion which exceeds the force from its parent. This condition is fulfilled during the collision when the internuclear distance becomes equal or smaller than the so-called release distance $R_{r}$ :

$$
R_{r}=\sqrt{q \cdot a \cdot a_{0}} \cdot v_{0} / v
$$

$a_{0}$ and $v_{0}$ are the Bohr radius and the Bohr velocity, respectively, whereas $a$ and $v$ are the orbit radius and velocity respectively of the bound electron [30]. Considering only outer electrons in neutrals or low charged ions, that yields roughly $\mathrm{R}_{\mathrm{r}} \approx \sqrt{\mathrm{q}} \cdot \mathrm{a}_{0}$. The released electron is normally captured by the ion because of its rather low velocity inside an EBIS. If the ion collides with another ion with a charge state $q_{2}$, the coulomb repulsion limits the closest approach in a head-on collision to

$$
R_{i i}=2 \cdot q \cdot q_{2} \cdot E_{o} / E^{\prime} \cdot a_{o}
$$


where $E^{\prime}$ is the sum of the kinetic energy of both ions in the center-of-mass system. Obviously only low charged ions in energetic collisions with small impact parameters come close enough to allow for the transfer of electrons, whereas collisions among low energy, highly charged ions are purely elastic.

Therefore, we conclude that the ion-ion collisions encountered by the highly charged ions inside an EBIS yield only very little recombination.

No coulomb repulsion limits the closest approach in ion-neutral collisions, which has been a center of interest for many years. Many collision systems have been investigated in great detail over a large energy range. It was found that the low energy collis ons are dominated by electron capture, and that collisions of highly charged ions with many-electron neutrals have a significant amount of multiple electron capture, even at very low energies [31]. The cross section for single electron capture is roughly $10^{-15} \cdot \mathrm{q} \mathrm{cm}^{2}$, approximately proportional to the charge state, but independent of the collision energ: in the low energy range $[32,33]$. Multiple electron capture reaches the same order of magnitude for very high charge states $[32,33]$. However, we would like to point out that only a very few experiments used charge states higher than 8 [34-37], most of which used EBISes for the ion production [34-36]. In addition, we are not aware of any experiments involving ver, highly charged ions at energies below $1 \mathrm{kV}$, despite the fact that one could expect an increase in cross section at very low collision energies due to the polarization of the neutral by the highly charged ion. 
The recombination of ions counteracts the ionization rate, which is decreasing with invreasing charge state. The ultimate equilibrium is reached when the ionization rate is matched by the recombination rate:

$$
n_{q-1} \cdot j / e \cdot \sigma_{i}(q-1, q)=n_{q}\left(R_{b \theta}+R_{s \theta}+R_{i}+R_{n}\right)
$$

where $R_{b e}$ is the rate of radiative and dielectronic recombination with beam electrons; $R_{\text {se }}$ is the rate of recombination with secondary electrons; $R_{j}$ is the rate of recombination from collisions with other ions; and $R_{n}$ is the rate of recombination from collisions with neutrals. Neglecting $R_{s e}$ and $R_{i}$, as discussed above, we obtain

$$
\begin{aligned}
& n_{q-1} \cdot j / e \cdot \sigma_{i}(q-1, q) \\
& \left.\quad=n_{d}\left(\sigma_{r I}(q, q-1)+\sigma_{d r}(q, q-1)\right) \cdot j / e+\sigma_{c}(q, q-1) n_{n} \cdot v_{j}\right)
\end{aligned}
$$

where $\sigma_{r r}$ and $\sigma_{d r}$ are the cross sections for radiative and dielectronic recombination with the beam electrons, $\sigma_{c}$ the cross section for capture from neutrals, $n_{n}$ the density of neutrals, and $v_{i}$ the mean velocity of the ions. This formula shows how the resonant dielectronic recombination cross section can be evaluated from the ratio of charge states if one subtracts the nonresonant background. If we avoid the dielectronic recombination and also neglect the very small radiative recombination, we obtain

$$
n_{q-1} \cdot j / e \cdot \sigma_{i}(q-1,1)=n_{q} \cdot \sigma_{c}(q, q-1) n_{n} \cdot v_{i}
$$


which often determines the maximum charge state which can be obtained with an EBIS. Having determined the current density and the ionization cross section, estimating the capture cross section and the mean ion velocity, and measuring the yields of the equilibrium charge states, we can estimate the density of the neutrals dixing operation. Hence, the useful range of confinement times is limited by the residual gas pressure inside the EBIS during operation, and the only way to reach higher charge states is by increasing the current density or by reducing the pressure inside the source, if the beam energy is adequate for ionization. For this reas. the second generation EBISes adapted high field solenoids to achieve high current densities and adapted cooling the trap to cryogenic temperature to reduce the pressure inside the trap, which can be simultaneously accomplished inside the cold bore of a superconducting solenoid. However, there is another important limitation which is discussed in the next section.

\section{Ion Heating}

The ions are trapped inside the source by the magnetic field and the linear net space charge $\mathrm{N}^{-}$of the electron beam, partially compensated by the trapped ions. If charge density inside the electron beam with radius $r_{0}$ is uniform, and the electron beam is going through the center of a drift tube with an internal radius $R$ and a voltage $U_{d t}$, the radial potential $U(r)$ is 


$$
\begin{aligned}
& U(r)=U_{d t}+2 \cdot N^{-} \cdot \ln (R / I) \quad\left(I>I_{0}\right) \\
& U(r)=U_{d t}+N^{-} \cdot\left(1-I^{2} / r_{0}^{2}+2 \cdot \ln \left(R / I_{0}\right)\right)
\end{aligned}
$$

The ions oscillate radially inside this potential well, whereas the magnetic field will cause the ion trajectories to rotate, forming beautiful figures [38]. The maximum radial excursion is determined by the ion energy. If its energy starts to exceed $\mathrm{q} \cdot \mathrm{N}^{*}$, the ion will leave the electron beam for an increasing fraction of time, and therefore decrease the apparent ionization w tor $j \cdot t$. If its energy keeps increasing, the ions may eventually hit a drift tube and recombine. In the axial direction, the trap is formed through the increased voltage on one drift tube at each end. As (9) applies to all drift tubes, the axial end barriers have the shape of a saddle point. The depth of the potential well $\delta U$, the height of the saddle point over the bottom of the well, is the voltage difference of the drift tubes corrected for the different depths of the space charge well which change with the local electron energy. In the axial direction the ions bounce back and forth until they have sufficient energy to climb over the end barrier.

If the ion is ionized when it is not at the bottom of the potential well (center of the electron beam away from the end barriers) or during an ion-ion collision, the ion gains energy equivalent to the product of its change of charge and the excess potential, and therefore the average ion energy is increasing 
with each ionization. However, the increase in charge state causes also the apparent depth of the potential well $q \cdot \delta U$ to increase and therefore, the average radial excursion is actually decreasing whenever the charge state is increasing $[38,39]$. The situation is reversed for recombination during ion-neutral collisions except that the Coulomb explosion following the charge transfer provides the ions with additional energy.

The main contribution of ion heating comes from the constant kombardment with eriergetic electrons, which transfer energy to the ions in every collision, including the abundant non-ionizing. The heating of ions by elastic collisions with electrons was originally formulated by spitzer [40], and it is interesting to note that the heating rate increases with the square of the ion's chargi state. Becker [41] made an approximation which reflects the EBIS geometry, and calculated the axial and the radial heating rate integrated over the ionization factor required to reach a certain charge state. He found that the energy gain may cause some ions to leave the trap before the highest charge states are reached $[A 1]$.

There is no coubt that the ion heating can cause a substantial loss of ions as one can see in Figure 7 [from 16], which shows a charge state evolution measured with the KSU-CRYEBIS. The figure shows also the sum of all ions, which indicates an enhanced loss for confinement times over two seconds. Naturally the discovery of ion cooling brought a relief to the EBIS community $[20,42,43]$. 


\section{v. Ion cooling}

We mentioned before that ion-ion collisions contribute very little to recombination. Sut when a low charged ion with a charge state $q_{2}$ collides with a highly charged ion, which is subject to a higher heating rate, energy is transferred and the highly charged ion is cooled, which coined the expression "ion-ion cooling" [7]. The low charged ion heats up until it overcomes its apparent trapping potential $q_{2} \cdot \delta U$, which is much smaller than the apparent trapping potential of the highly charged ion. "Evaporative cooling" is the other expression, which reflects the important fact that the low charged ions remove energy when they leave the trap $[20,43]$. Hence, continuous cooling requires a constant addition of cold low charged ions to replace the evaporated ones. The question arises how and which coolant should be introduced for efficient cooling. It is quite simple to bleed neutrals into the trap $[20,43,44]$, hoping that the gas will be ionized pretominantly by the electron beam. The technique of gas mixing [45] or the use of molecular species [7] can also be employed. However, if the radial excursions of the highly charged ions exceed the electron beam radius, the ion-neutral recombination may become important. We discussed above that reco. bination can also play a role in collisions with low charged ions. Hence injecting ions will minimize, but not necessarily eliminate recombination. If ions are injected over the end barrier [46], only a charge changing collision would trap the cooling ions, which favors very low charge states for injection. A drawback of this very simple 
scheme is that the injected ions are not very cold. A continuous injection of ions circumvents the trapping requirement and allows for the injection of higher crirge states to minimize recombination. However producing highly charged ions can be an additional complication. Cold ions can be introduced through a load lock system [7]. The electron beam flooding the lock automatically increases the charge states of the cooling ions and therefore minimizes recombination. The mass and the maximum charge state of the cooling species affects the rate of cooling and recombination, which seems to favor intermediate masses $[20,44,47]$. On the other hand, the reinjection of the production species replaces the lost ions with the ones which reach high charge states before they get too hot $[7,46]$.

Ion cooling is not quite new, as the residual gas provided natural cooling all along, a fact supported by much evidence $[20,43,44,48-50]$. However, the recognition of ion-ion cooling led to active cooling which increases the ion yields, produces higher charge states, or decreases required confinement time. Most of the schemes discussed above have been tested successfully, and increases in ion yields up to a factor of 20 have been reported [6]. However, with so many different schemes, different cooling species, and different devices, it will take some time to gain experience, to sort out the results and to get a handle on the technical know-how. We have to keep in mind that the system is rather complex, as it involves recombination of the highly charged ions, the apparent ionization factor, and containment of the production species, all due to the 
interactions of the electron beam with the low charged and the highly charged ions, in addition to the interaction between the ion species. However, we can expect to get some help and directions from computer models, which takes all these processes into consideration $[47,51]$.

\section{vI. Conclusions}

We have discussed the processes which play a role in the production of highly charged ions with Electron Beam Ion Sources. Ionization- and recombination processes have been sufficiently known for a long time, although recent experiments provided more jnsight into recombination processes. The effect of ion heating also was recognized a lon: time ago, but did not get much attention until the cooling effect in collisions with low charged ions was recognized. However, there are many ways to introduce the ions needed for cooling, and many are still experimenting to find the most exficient way. This effort will reduce the losses during long confinement needed for the ionization of the highest charge states and thus improve the production yields.

\section{Acknowledgments}

Supported by the Division of Chemical sciences, office of Basic Energy Sciences, Office of Energy Research, U.S. Department of Energy. 


\section{References}

[1] E.D. Donets, USSR Inventor's Certificate No. 248860, (March 16, 1967), BYull. OIPOTZ No. 23, (1969) 65.

[2] Martin P. Stockli, submitted for publication in zeitschrift fuer Physik D (1991).

[3] L. Schneider, M.W. Clark, D. Dewitt, R. Schuch, C.L. Cocke, R. Schmieder, K. Reed, R. Marrs, M. Levine, R. Fortner, 12th Int. Conf. on Atomic Physics, Ann Arbor, MI, USA, Abstract of Contributed Papers (1990) II-1.

[4] D. Schneider, 5th Int. Conf. on the Physics of Highly Charged Ions, Giessen, Germany, (1990).

[5] E.D. Donets, in: The Physics and Technology of Ion Sources, ed. I.G. Brown, (John Wiley \& Sons, New York, 1989) p. 245.

[6] E.D. Donets, Int. Conf. on Ion Sources, Berkeley, CA, USA, (1989) .

[7] E.D. Donets, Rev. Sci. Instrum. 61 (1990) 225.

[8] A. Hershcovitch, ed., International Symposium on Electron Beam Ion Sources and Their Applications, Upton, NY, USA, 1988, AIP Conf. Proc. No. 188, (American Institute of Physics, New York, 1989).

[9] I.G. Brown ed., International Conference on Ion Sources, Berkeley, CA, USA, 1989, Rev. Sci. Instrum. 61 (1990) $221-666$.

[10] Martín P. Stockli, J. Arianer, C.L. Cocke, and P. Richard, Nucl. Instr. and Meth. B40/41 (1989) 1020.

[11] M.A. Levine, R.E. Marrs, C.L. Bennett, J.R. Henderson, D.A. Knapp, M.B. Schneider, in Ref. [8], (1989) 82 . 
[12] J. Arianer, A. Cabrespine, and C. Goldstein, Nucl. Instr. and Meth. 193 (1982) 401 .

[13] e.g. D.C. Gregory, M.S. Huq, F.W. Meyer, D.R. Swenson, M. Sataka, and S. Chantrenne, Phys. Rev. A $\underline{41}$, (1990) 106.

[14] e.g. A. Muller, C. Achenbach, E. Salzborn, and R. Becker, J. Phys B 17 (1984) 1427 .

[15] Alfred Mulier, Phys. Lett. 113A (1986) 415.

[16] C.L. Cocke, accepted for publication in Nucl. Instr. Meth. (1991)

[17] E.D. Donets, and A.I. Pikin, Sov. Phys. JETP. $\underline{43}$ (1976) 1057.

[18] E.D. Donets, and V.P. Ovsyannikov, Sov. Phys. JE.?. $\underline{53}$ (1981) 466 .

[19] R.W. Schmieder, in: Physics of Highly-Ionized Atoms, ed. R. Marrus, (Plenum Publishing Corporation, 1989) p. 321.

[20] M.A. Levine, R.E. Marrs, J.R. Henderson, D.A. Knapp and M.B. Schneidor, Physic'a Scripta. T22 (1988) 157.

[21] H. Bethe, and E. Salpeter, in: Atome I, ed. S. Flugge, Handbuch der Physik, Vol35 (Springer Verlag, Berlin, 1957)

[22] R.E. Marrs, M.A. Levine, D.A. Knapp, and J.R. Henderson, Phys. Rev. Lett. 60 (1988) 1715.

[23] L.H. Andersen, J. Bolko, and P. Kvistgaard, Phys. Rev. Lett. 64 (1990) 729 .

[24] R.E. Marrs, P. Beiersdorfer, C. Bennett, M.H. Chen, T. Cowan, D. Dietrich, J.R. Henderson, D.A. Knapp, A. osterheld, M.B. Schneider, and J.H. Scofield, in Ref. [8] (1989) 445 . 
[25] R. Ali, C.P. Bhalla, C.L. Cocke, M. Schulz, and M. Stockli, submitted for publication in Zeitschrift fuer Physik D (1991).

[26] R. Ali et al. to be submitted for publication in Phys. Rev. A. (1990) .

[27] D.A. Knapp, R.E. Marrs, M.A. Levine, C.L. Bennett, M.H. Chen, J.R. Henderson, M.B. Schneider, and J.H. Scofield, Phys. Rev. Lett. $\underline{62}$ (1989) 2104.

[28] R. Ali, C.P. Bhalla, C.L. Cocke, and M. Stockli, Phys. Rev. Lett. $\underline{64}(1990) 633$.

[29] M. Schulz, R. Ali, C.L. Cocke, S. Hagmann, M. Stockli, and H. Schmidt-Böcking, this volume (1991).

[30] N. Bohr, and J. Lindhard, K. Dan. Vidensk. Selsk. Mat. Fys. Medd. 28 , No.7 (1954).

[31] C.L. Cocke, R. DuBois, T.G. Gray, E. Justiniano, and C. Can, Phys. Rev. Lett. $\underline{46}$ (1981) 1671.

[32] A. Muller and E. Salzborn, Phys. Lett. 62A (1977) 391.

[33] L. Liljeby, G. Astner, A. Barany, H. Cederquist, H. Danared, S. Huldt, P. Hvelplund, A. Johnson, H. Knudsen, and K.G. Rensfelt, Phys. Scripta $\underline{33}$ (1986) 310 .

[34] H. Tawara, ed., The collected Papers of NICE Project/IPP, Nagoya, IPP-AM-43, (Institute of Plasma Physics, Nagoya University, 1985).

[35] H. Andersson, G. A.stner, and H. Cederquist, J. Phys B21 (1988) L187.

[36] H. Cederquist, H. Andersson, G. Astner, P. Hvelplund, and J.O.P. Pederson, Phys. Rev. Lett. $\underline{62}$ (1989) 1465 . 
[37] J.P. Briand, P. Charles, S. Essabaa, M. Mayo, S. Bliman, P. Briand, and R. Geller, XVth Int. Conf. on the Physics of Electronic and Atomic Collisions, Brighton, UK, Abstracts of Contributed Papers (1987) 564.

[38] R.W. Schmieder, in: Proceedings of the Workshop on opportunities for Atomic Physics using slow, Highly-Charged Ions, (Argonne National Laboratory, ANL-PHY-87-1, 1987) p. 253 .

[39] R.W. Schmieder, Physica Scripta. T22 (1988) 312.

[40] L. Spitzer, Physics of Fully Ionized Gases, (Interscience, New York, 1956)

[41] R. Becker, in: II EBIS Workshop, eds. J. Arianer and $M$. Olivier (Institut de Physique nucleaire, Orsay, France, 1981) p. 185

[42] E.D. Donets and G.D. Shirkov, Avtorskoe Svidetelstvo USSR, N1225420 (1984), and Bul. OI, N44 (1989) 69.

[43] M.A. Levine, M.B. Schneider, in: Proceedings of the International Conference on ECR Ion Sources, East Lansing, 1987, National Superconducting Cyclotron Laboratory, Michigan State University Report MSUCP-47 (1988) 233.

[44] M.B. Schneider, M.A. Levine, C.L. Bennett, J.R. Henderson, D.A. Knapp, and R.E. Marrs, in Ref. [8], (1989) 158.

[45] R. Becker and M. Kleinod, 5th Int. Conf. on the Physics of Highly Charged Ions, Giessen, Germany, (L990) and to be published in Zeitschrift fuer Physik D (1991).

[46] J. Faure, private communication (1989). 
[47] 5.M. Penetrante, M.A. Levine, and J.N. Bardsley, in Ref. [8] (1989) 145 .

[48] E.N. Beebe, in Ref. [8] (1989) 166.

[49] R.W. Schmiєder, C.L. Bisson, S. Haney, N. Toly, A.R.Van Hook, and J. Weeks, in Ref. [8] (1989) 45.

[50] R.W. Schmieder and C.L. Bisson, Rev. Sci. Instrum. 61 (1990) 256 .

[51] R.W. Schmieder and C.L. Bisson, unpublishod (1989). 


\section{Figure Captions}

Fig. 1: Schematic of an Electron Beam Ion Source with the cathode (1) and the anode (2) of its electron gun, the solenoid (3) with its magnetic shield (4), the collector (5) with its repeller (6) and the drift tubes (7). The electron beam (8) is absorbed by the collector whereas the ion beam (9) is extracted by the repeller. Fig. 2: Theoretical charge state evolution of Argon bombarded with $10 \mathrm{kV}$ electrons as a function of the ionization factor $j \times t$ [from Ref. 12].

Fig. 3: Measured charge state distribution of Argon bombarded with $5.4 \mathrm{kV}$ electrons for $0.04,0.33,0.63$ and 2.33 seconds [from Ref. 16].

Fig. 4: Theoretical (curve) and experimental (circles) cross section for dielectronic recombination of heliumlike Argon as a f. nction of the electron energy [from Ref. 26].

Fig. 5: Equilibrium ion yields (after confinement of 2 seconds) for $A r^{14+}, A r^{15+}$, and $A r^{16+}$ as a function of the electron beam energy. Whenever the electron beam energy is in resonance with a doubly excited state of $\mathrm{Ar}^{15+}$, the $\mathrm{Ar}^{15+}$ yield will increase through dielectronic recombination of the $\mathrm{Ar}^{16+}$. The top line shows the sum of the three charge states [from Ref. 26].

Fig. 6: Theoretical (curve) and experimental (dots) differential $x$-ray production cross section of Argon as a function of the electron beam energy [from Ref. 26].

Fig. 7: Measured charge state evolution of Argon bombarded with a $5.4 \mathrm{kV}$ electron beam as a function of containment time [from Ref. 16]. 


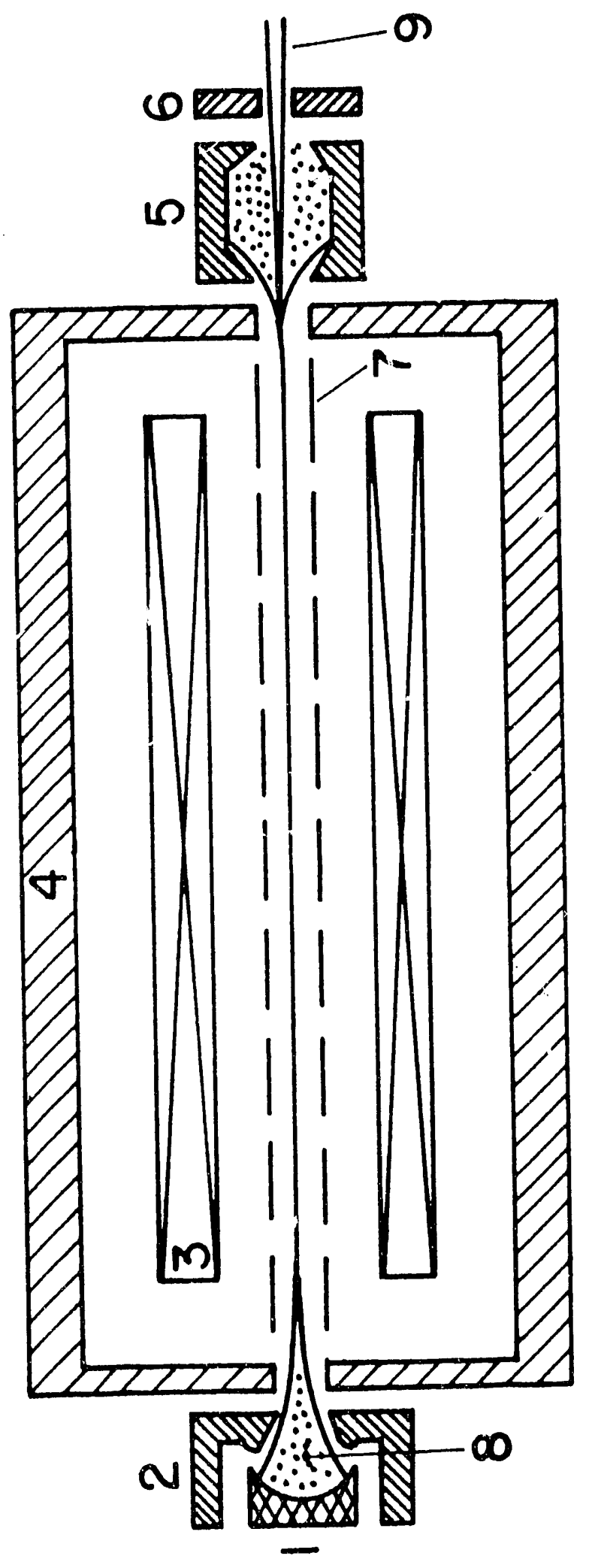




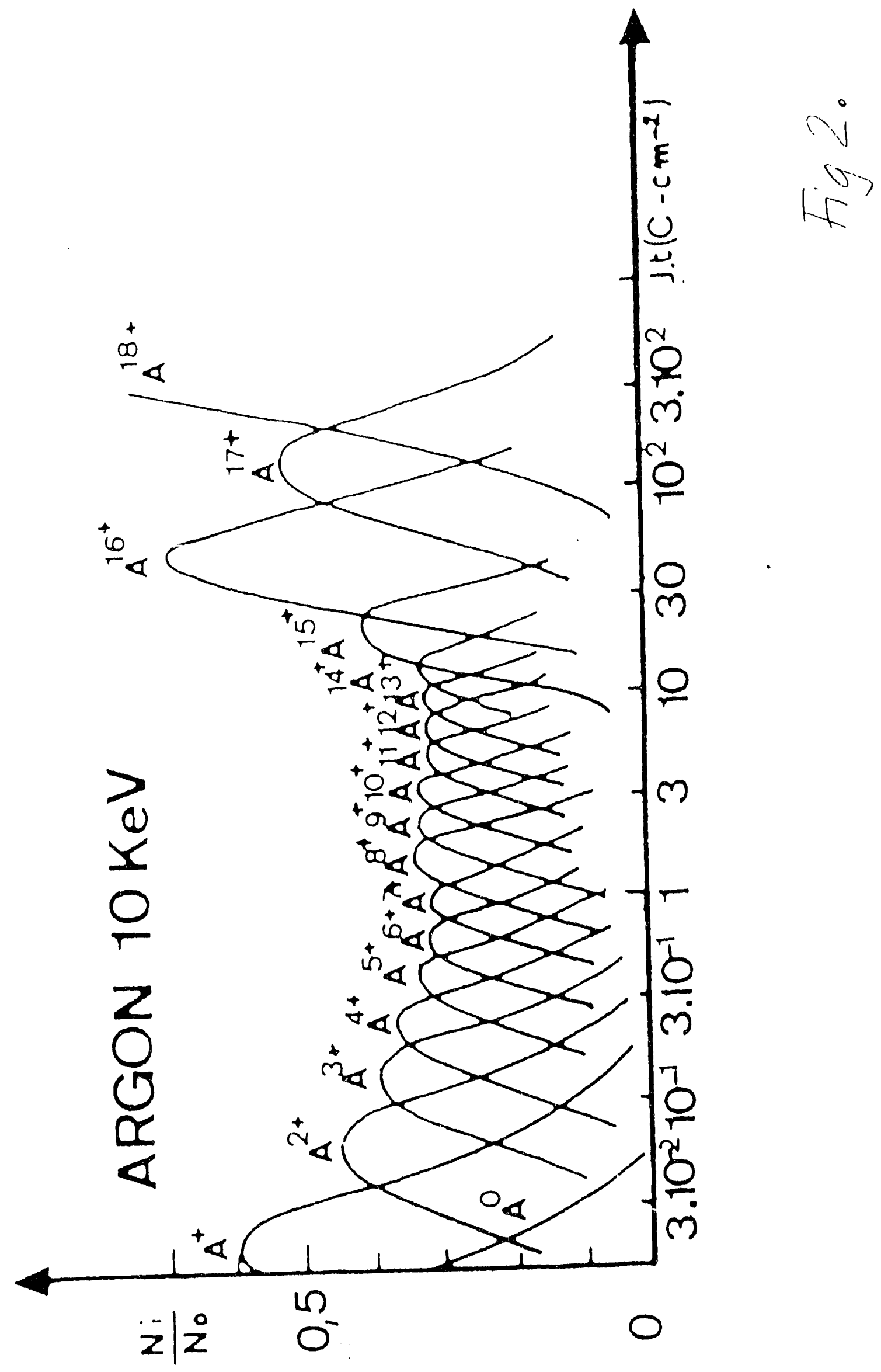


.

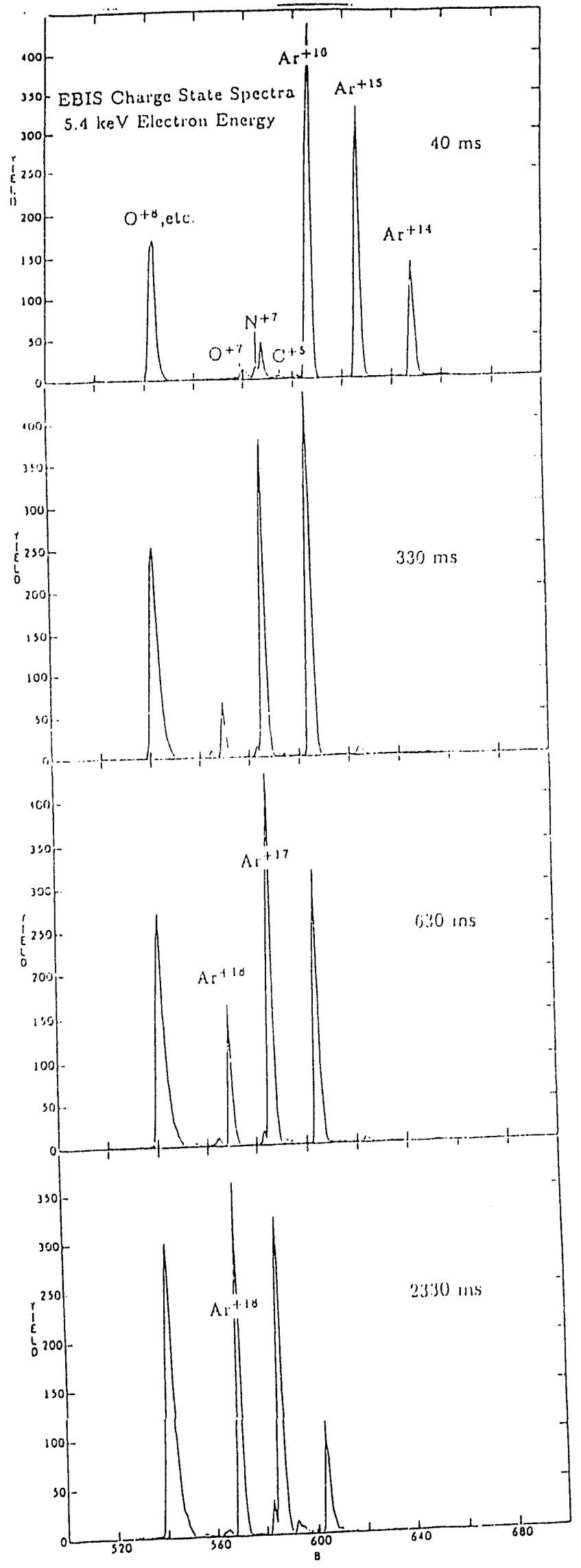

Fig 3 
.

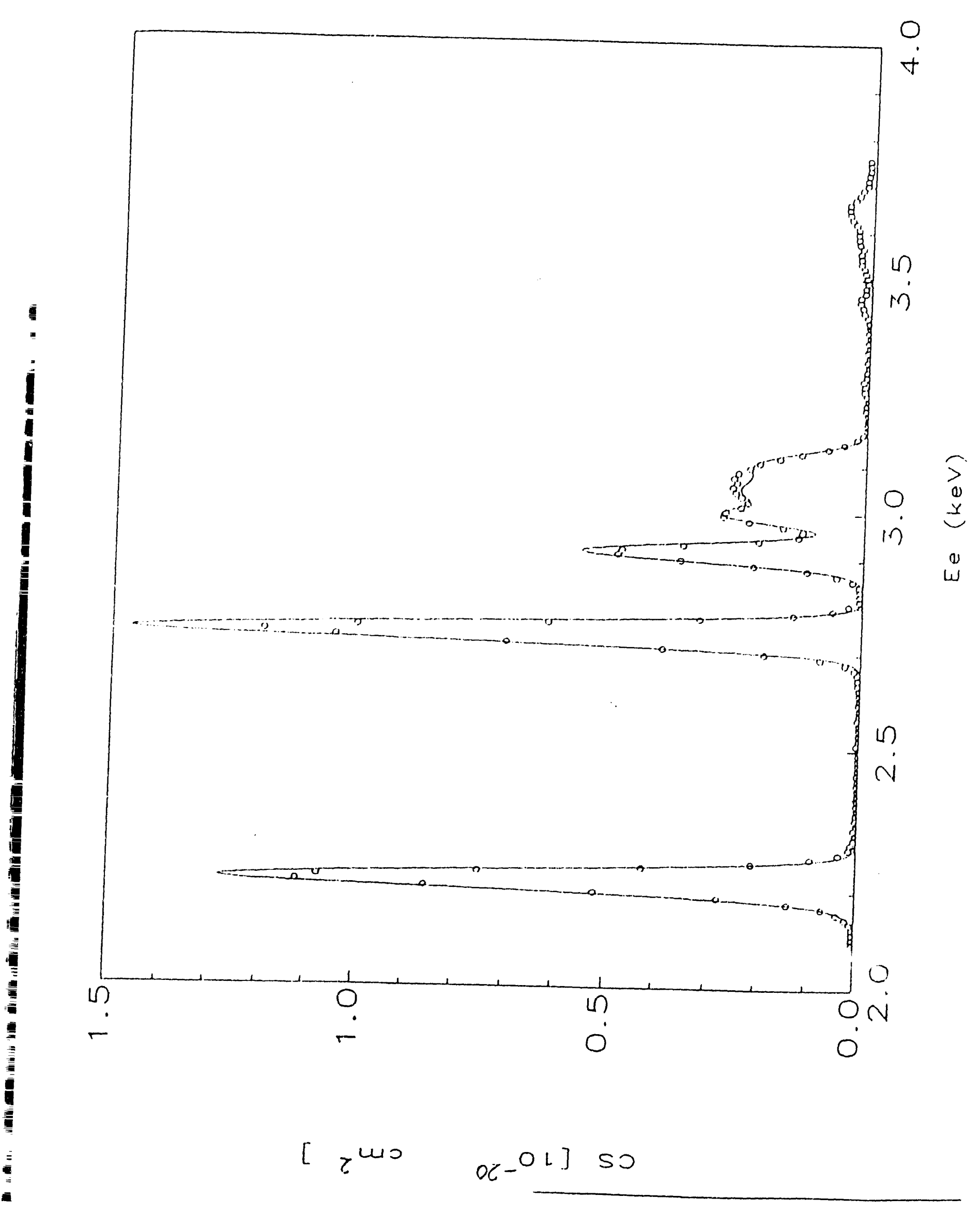




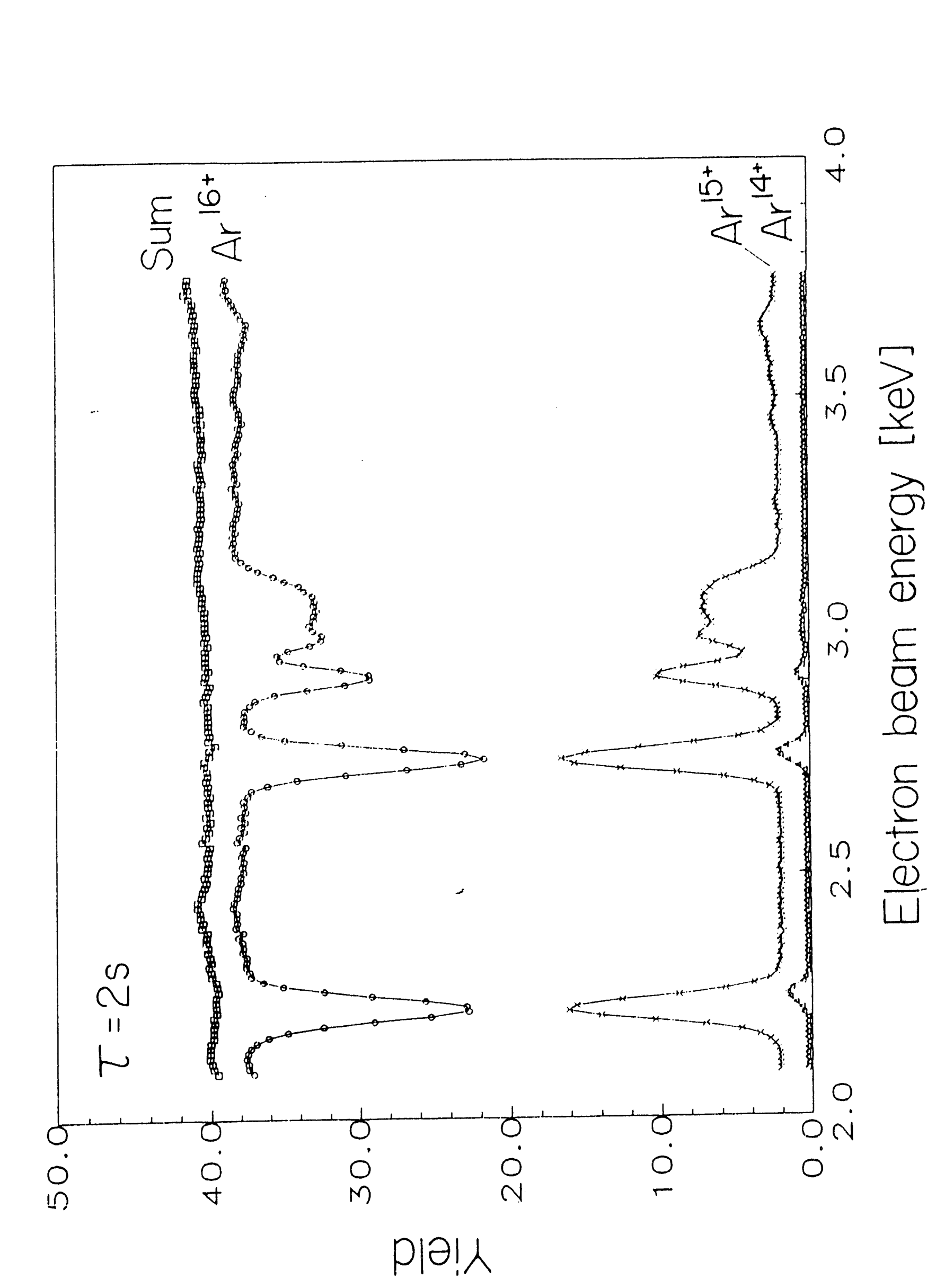

$|-i\rangle$

15 


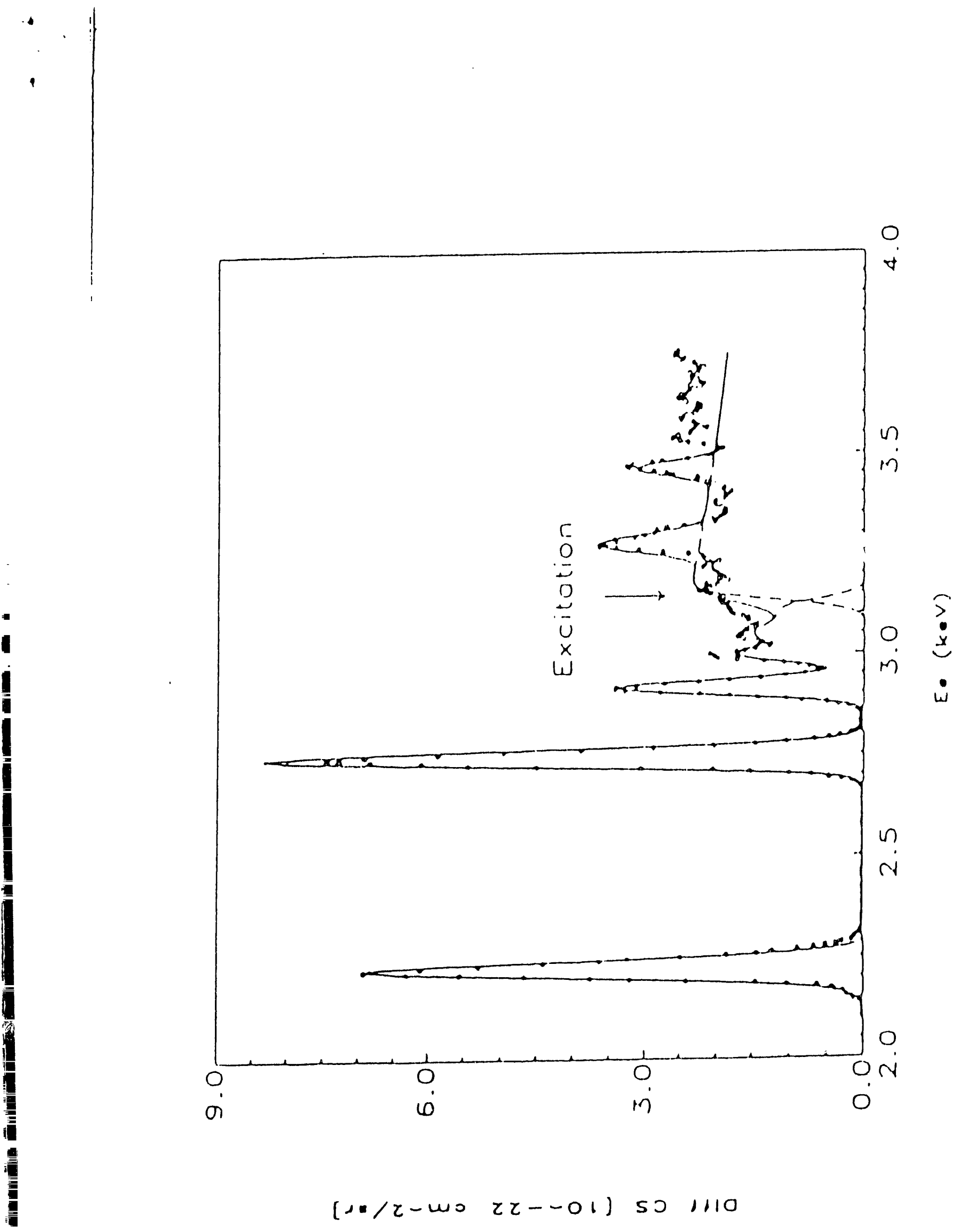

$\because n$ $45^{5}$ 


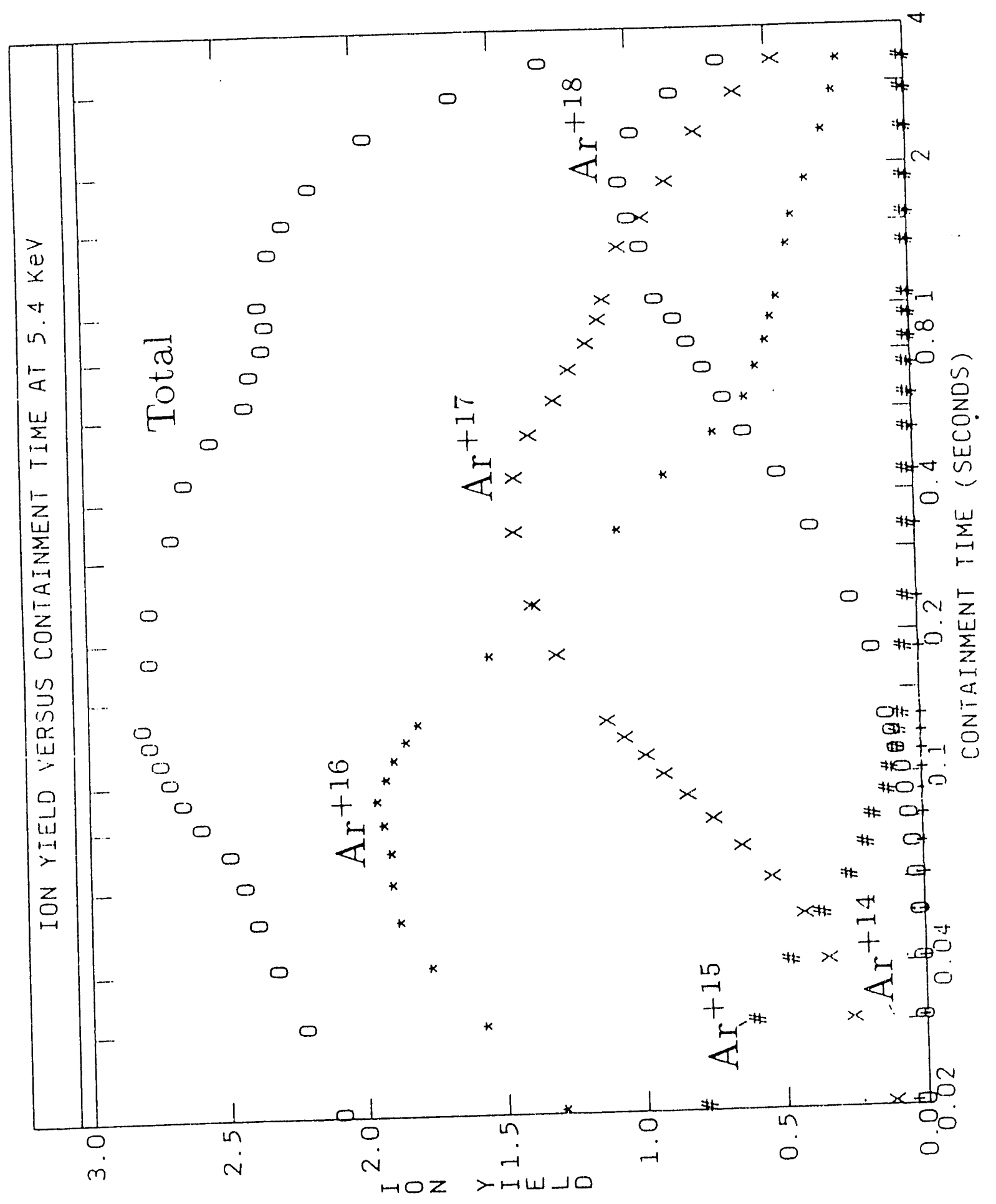



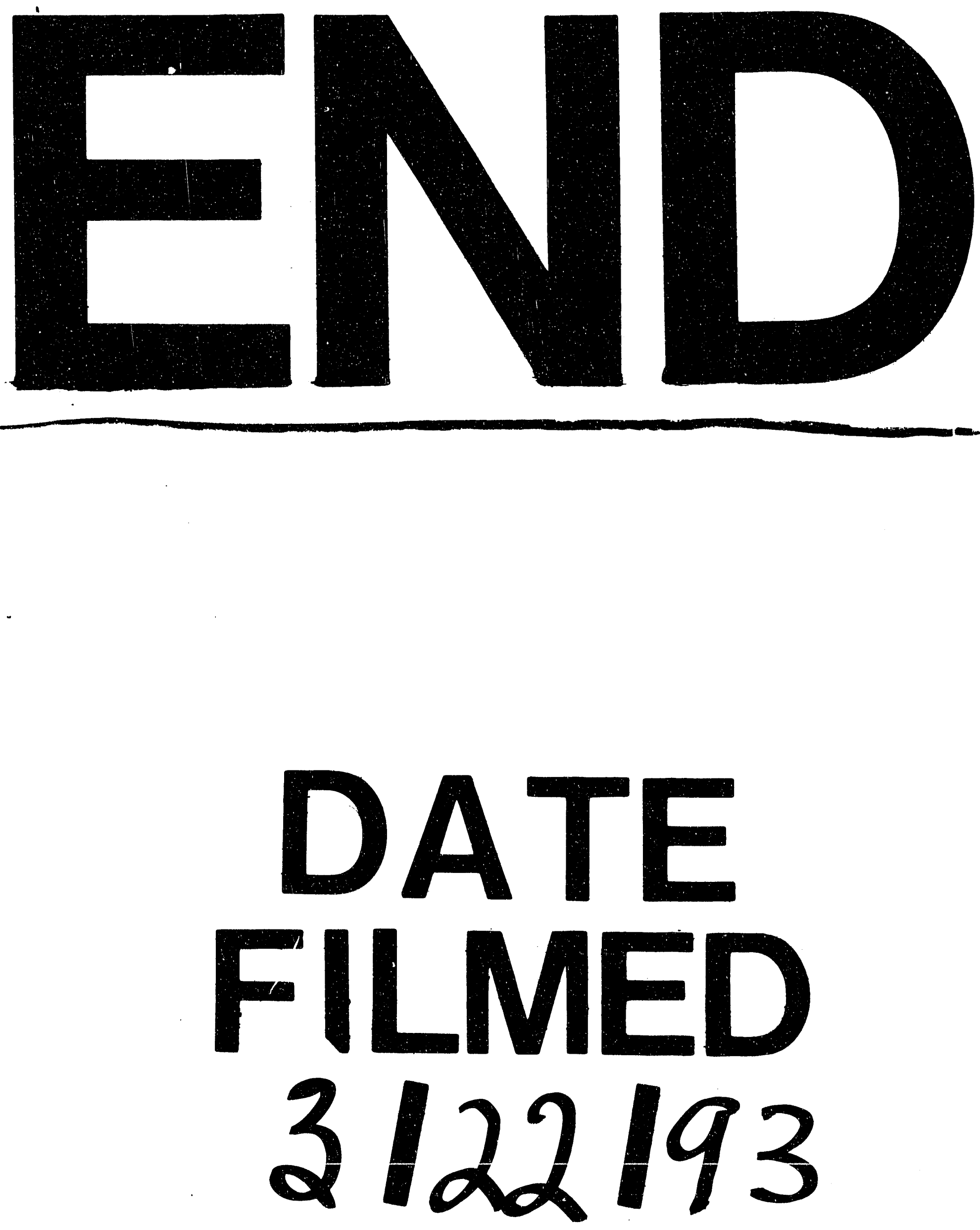


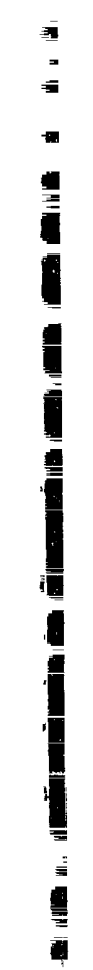

